\title{
The Assessment of Reperfusion Inflammatory Injury in Ischemic Preconditioned Diabetic Rats
}

\author{
Grigorescu Bianca-Liana¹, Fodor Raluca Ștefania²*, Scridon Alina ${ }^{3}$, Perian Marcel ${ }^{3}$, Badea ludita², Cioc \\ Adrian Dan¹, Cotoi Ovidiu Simion4, Copotoiu Sanda-Maria², Azamfirei Leonard² \\ Department of Anaesthesiology and Intensive Care, Emergency County Hospital, Tirgu-Mures, Romania \\ 2 Department of Anaesthesiology and Intensive Care, University of Medicine and Pharmacy, Tirgu-Mures, Romania \\ 3 Department of Physiology, University of Medicine and Pharmacy, Tirgu-Mures, Romania \\ 4 Department of Pathophysiology, University of Medicine and Pharmacy, Tirgu-Mures, Romania
}

\begin{abstract}
Objective: The assessment of systemic reperfusion injury and the contractile force of the peripheral muscles post-acute ischemia of the hind limbs in healthy versus diabetic ischemic preconditioned rats. Method: The study included 16 Wistar rats divided into two groups: the control group and the diabetic ischemic preconditioned group. Acute ischemia was induced, followed by reperfusion. The assessment of reperfusion injury used biochemical, histopathological and functional determinations (peak tetanic tension-PT, specific tension-ST). Results: Ischemiareperfusion injury was more severe in control group regarding creatine-kinase (CK) (CK1=470.13 IU/L versus CK2=230.88 IU/L, $p=0.0001)$ and myoglobin $(390.25 \mathrm{ng} / \mathrm{mL}$ versus $47.99 \mathrm{ng} / \mathrm{mL}, \mathrm{p}=0.025)$. Cytolysis enzymes were significantly increased in diabetic preconditioned rats (Alanine aminotransferase ALAT1=46 IU/L, ALAT2=167.8 IU/L, $p=0.02$; Aspartate aminotransferase ASAT1=106 IU/L, ASAT2=237.5 IU/L, $\mathrm{p}=0.016$ ). Functional assessment (PTT and ST) highlighted roughly equal values. A paradoxical response occurred in diabetic rats (the contractile force increased during the period of the stimulation). Histopathological findings showed that rhabdomyolysis was more severe in the control group, while inflammatory systemic response due to reperfusion injury was less expressed in diabetic ischemic preconditioned rats. Conclusions: Ischemic preconditioning reduces the severity of reperfusion injury and allows the preservation of contractile muscle function in diabetic rats.
\end{abstract}

Keywords: reperfusion injury, ischemic preconditioning, contractility, diabetes, skeletal muscle

Received: 04 August 2015 / Accepted: 14 August 2015

\section{Introduction}

Diabetes mellitus involves multiple micro-and macro vascular complications, that rapidly and irreversibly aggravates the morphofunctional status of various organ and systems, as well as the hind limbs. In most cases, revascularization techniques are associated with local and systemic complications secondary to ischemia-reperfusion injury (IRI). The duration and severity of ischemia, as well as the promptness and the type of revascularization technique, determine the evolution towards either rapid biochemical, structural and functional recovery, or the total irreversible damage of the ischemic area. Reperfusion induces a systemic inflammatory response, secondary to the activation of macrophages and the release of oxygen free radicals into the bloodstream, which cause endothelial damage and the release of tissue injuring proinflammatory cytokines $[1,2]$.

Ischemic preconditioning (IP) is the most common and most effective non-invasive technique that provides protection against IRI by inducing short periods of ischemia followed by reperfusion in a specific territory $[1,3,4]$. Saita et al demonstrated that the effectiveness of pre-conditioning is related to the duration and not to the number of cycles applied. The best results are achieved when applying 10 minutes ischemia and 10 minutes reperfusion for three cycles each [5]. Fernandez et al demonstrated that the benefit of these method lies in the anti-inflammatory effect. This effect occurs post IP in the hind limbs, due to reduced conversion of xanthine dehydrogenase $(\mathrm{XDH})$ to xanthine oxidase $(\mathrm{XOH})$ and the release of reactive oxygen species (ROS), decreased neutrophils infiltration and oxidative stress [6].

IP presents a biphasic evolution pattern. The early phase (classic preconditioning) occurs within the first three hours of ischemia, being independent of protein synthesis. After 24 hours the second window onsets (the late phase), which lasts for 48 hours and is associated with protein expression in the vascular endothelium and increased adenosine synthesis. Adenosine induces vasodilatation, anti-aggregation of platelets, inhibits cell adhesion, and also regulates neutrophil functions. Thus adenosine stimulates the systemic protection of organs located distal to the area where ischemia occurred. Remote ischemic preconditioning (RIP) also increases the adenosine synthesis where in short-term ischemia of an organ followed by reperfusion provides distant organ protection against IRI. This technique is especially used in case of surgical treatment of the heart, lung, kidney and liver [3,7].

The objective of the study was to assess of the extent of ischemia-reperfusion injury following acute ischemia of the hind limbs in diabetic ischemic preconditioned rats. 


\section{Methods}

This study was approved by the Research Ethics Board of the University of Medicine and Pharmacy of Targu-Mures (Registration number 70/17.09.2014).

Our study included a number of 16 male Wistar rats, aged 8 weeks, weighing between 368 and 430g, randomly divided into two groups: group 1 ( $\mathrm{n}=8$, control group), including diabetic rats and group $2(\mathrm{n}=8)$ including diabetic ischemic preconditioned rats. The animals were acclimated to usual laboratory condition 14 days before the experiment (ambient temperature of $22^{\circ} \mathrm{C}$, circadian rhythm of light). The rats were fed with standard laboratory rodent food (provided by Cantacuzino Institute, Bucharest, Romania) and water ad libitum. In all studied rats diabetes was induced using intraperitoneal administration of Streptozotocin (Streptozotocin MixedAnomers, Sigma-Aldrich, Canada), $60 \mathrm{mg} / \mathrm{kg}$. The presence of diabetes was confirmed by clinical evidence (polyuria, polyphagia, polydipsia, weight loss) and by serial measurements of fasting glucose from day 4 after Streptozotocin administration. The blood samples were obtained after eight hours of fasting, by vena caudalis puncture. The cut-off values were considered to be greater than $15 \mathrm{mmol} / \mathrm{dL}$ or $270 \mathrm{mg} / \mathrm{dL}[8,9]$.

Four weeks after the onset of diabetes, subsequent to 12hour fasting, group 2 underwent ischemic preconditioning, in general anesthesia with intraperitoneal injection of Ketamine-Xylazine $(50 \mathrm{mg} / \mathrm{kg}+10 \mathrm{mg} / \mathrm{kg})$. We induced three cycles of 10 minutes of ischemia/reperfusion by applying a tourniquet on the upper part of the left hind limb. The efficiency of the induced ischemia was assessed clinically on the basis of capillary refill time ( $\geq 3$ seconds). After 24 hours post IP both groups underwent acute ischemia, under general anesthesia (by inhalation of Sevoflurane), by applying a tourniquet on the upper part of the left thigh for 20 minutes, followed by reperfusion for 30 minutes. In order to prevent ilio-femoral bundle thrombosis, we administered 250 IU of sodium heparin before clamping. Volume replacement was performed by intraperitoneal administration of $5 \mathrm{~mL} / \mathrm{kg}$ body weight of saline solution. Blood samples for laboratory assays were collected via terminal cardiac puncture, and samples of muscle, kidney and liver tissues were harvested for histopathological examinations. The extensor hallucis brevis muscles were carefully removed, weighed, and rapidly transferred into the Steiert organ bath. The muscles were connected to the force transducer, progressively pre-tensioned to $9.8 \mathrm{mN}$ of force, and allowed $30 \mathrm{~min}$ of equilibration under constantflow perfusion of oxygenated $\left(95 \% \mathrm{O}_{2}+5 \% \mathrm{CO}_{2}\right)$ KrebsHenseleit solution containing (in $\mathrm{mM}$ ) $118.0 \mathrm{NaCl}, 4.7$ $\mathrm{KCl}, 25.0 \mathrm{NaHCO}_{3}, 1.2 \mathrm{MgSO}_{4}, 1.25 \mathrm{CaCl}_{2}, 1.2 \mathrm{KH}-$ ${ }_{2} \mathrm{PO}_{4}$, and 11.0 glucose, at $37^{\circ} \mathrm{C}$. Then, electrical stimulation of the muscles was performed at $166 \mathrm{~Hz}$ to induce muscle tetanus using two trains of stimuli, $4 \mathrm{msec}$ and 1 sec long, respectively, separated by a $5 \mathrm{~min}$ period of rest.

For each of the two protocols, the following parameters were measured: the peak tetanic tension (PTT), calculated as the difference between the maximum amplitude of the isometric contraction induced at electrical stimulation and the baseline level; the velocity of the tetanic contraction $\left(\Delta \mathrm{T} / \Delta \mathrm{t}_{\mathrm{c}}\right)$; the specific tension (ST), calculated as the PTT / muscle weight; the velocity of muscle relaxation $\left(\Delta \mathrm{T} / \Delta \mathrm{t}_{\mathrm{r}}\right)$. The serums obtained were centrifuged $(2000 \mathrm{r} / \mathrm{min}$ for $3 \mathrm{~min})$ and analyzed using a Miura type analyzer. Microscopic analyses of the biopsy specimens were performed after standard processing, Hematoxylin-Eosin staining and images were analyzed by using a NIKONECLIPSEE-800 microscope with $2 \mathrm{x}, 4 \mathrm{x}, 10 \mathrm{x}, 20 \mathrm{x}, 40 \mathrm{x}$ objectives.

SPSS statistical software package version 20 was used for statistical analysis. Student's t-test (for normally distributed data), and Mann-Whitney U-test (for non-normally distributed data), were used for analysis, with the confidence interval set to $95 \%$, and a $\mathrm{p}$ value lower than 0.05 was considered statistically significant. All data were expressed as mean values $\pm S D$.

\section{Results}

The results of the biochemical evaluations (mean values, standard deviations and their statistical significance) are summarized and compared in Table I. Functional evaluation revealed no significant difference between the two groups. Peak tetanic tension (PTT), tetanic contraction velocity $(\Delta \mathrm{T} / \Delta \mathrm{tc})$; specific tension $(\mathrm{ST})$ and muscle relaxation velocity $(\Delta \mathrm{T} / \Delta \mathrm{tr})$ showed no statistically significant differences, although the mean contraction and relaxation velocity were higher and the duration of muscle relaxation after contraction was shorter than in the control group (Table II). Histopathological findings revealed ischemia and reperfusion injury at the level of the skeletal muscles. The lesions were more markedly expressed in the control group. Changes of the skeletal muscle represented by the fragmentation of fibers and rhabdomyolysis were observed in the control group, whereas areas of coagulative necrosis with the preservation of muscle fiber architecture were reported in IP rats.

Table I. The results of biochemical assessment after post-ischemic reperfusion in the control group and diabetic rats

\begin{tabular}{lccc}
\hline Parameters & $\begin{array}{c}\text { Control - diabetic } \\
(\mathrm{n}=8)\end{array}$ & $\begin{array}{c}\text { Diabetic \& } \\
\text { preconditioned } \\
(\mathrm{n}=8)\end{array}$ & p-value \\
\hline Blood glucose & $425.1 \pm 98.8$ & $420.7 \pm 74.6$ & 0.86 \\
$\begin{array}{l}\text { Myoglobin } 1(\mathrm{ng} / \\
\mathrm{mL})\end{array}$ & $390.25 \pm 322.05$ & $47.99 \pm 30.039$ & 0.025 \\
CK (IU/L) & $470.13 \pm 107.18$ & $230.88 \pm 51.90$ & 0.0001 \\
ASAT (IU/L) & $106 \pm 107.18$ & $237.50 \pm 112.69$ & 0.84 \\
ALAT (IU/L) & $46 \pm 6.59$ & $167.38 \pm 70.85$ & 0.02 \\
LDH (IU/L) & $884.88 \pm 451.36$ & $1320 \pm 387.80$ & 0.127 \\
Urea (mg/dL) & $57.89 \pm 7.61$ & $60.55 \pm 15.16$ & 0.717 \\
Creatinine $(\mathrm{mg} / \mathrm{dL})$ & $0.71 \pm 0.16$ & $0.63 \pm 0.22$ & 0.103 \\
\hline CK & & & \\
\hline
\end{tabular}

$\mathrm{CK}=$ creatinekinase, ASAT=aspartate aminotransferase, ALAT=alanine aminotransferase, $\mathrm{LDH}=$ lactate dehydrogenase 
Table II. Mechanical parameters measured in the two groups

\begin{tabular}{lccc}
\hline Parameters & $\begin{array}{c}\text { Control - diabetic } \\
(\mathrm{n}=8)\end{array}$ & $\begin{array}{c}\text { Diabetic \& } \\
\text { preconditioned } \\
(\mathrm{n}=8)\end{array}$ & p-value \\
\hline $\begin{array}{l}\text { Muscle weight } \\
(\mathrm{mg})\end{array}$ & $45.44 \pm 8.55$ & $48.66 \pm 14.62$ & 0.59 \\
\hline Stimulation 1 & $4.95 \pm 2.91$ & $5.33 \pm 2.74$ & 0.79 \\
$\mathrm{PTT}(\mathrm{mN})$ & $25.51 \pm 11.80$ & $26.74 \pm 10.46$ & 0.84 \\
$\Delta \mathrm{T} / \Delta \mathrm{tc}(\mathrm{mN} / \mathrm{sec})$ & $0.11 \pm 0.08$ & $0.12 \pm 0.09$ & 0.75 \\
$\mathrm{ST}(\mathrm{N} / \mathrm{g})$ & $20.32 \pm 14.40$ & $21.97 \pm 13.21$ & 0.83 \\
$\Delta \mathrm{T} / \Delta \operatorname{tr}(\mathrm{mN} / \mathrm{sec})$ & & & \\
\hline $\mathrm{Stimulation} 2$ & $5.46 \pm 2.56$ & $4.36 \pm 1.93$ & 0.36 \\
$\mathrm{PTT}(\mathrm{mN})$ & $18.64 \pm 12.95$ & $16.68 \pm 18.13$ & 0.49 \\
$\Delta \mathrm{T} / \Delta \mathrm{tc}(\mathrm{mN} / \mathrm{sec})$ & $0.12 \pm 0.07$ & $0.11 \pm 0.08$ & 0.56 \\
$\mathrm{ST}(\mathrm{N} / \mathrm{g})$ & $13.56 \pm 8.59$ & $9.84 \pm 5.42$ & 0.34 \\
$\Delta \mathrm{T} / \Delta \operatorname{tr}(\mathrm{mN} / \mathrm{sec})$ & & &
\end{tabular}

Stimulation 1 refers to the muscle response to the $4-\mathrm{msec}$ train of stimuli. Stimulation 2 refers to the muscle response to the $1-\mathrm{sec}$ train of stimuli. PTT - peak tetanic tension; $\Delta T / \Delta t_{c}$ - velocity of contraction; $S T$ - specific tension; $\Delta \mathrm{T} / \Delta \mathrm{t}_{\mathrm{r}}$ - velocity of relaxation.

There were more significant structural changes in the control group consisting by the presence of eosinophilic precipitate within the renal tubules (precipitated myoglobin). The main kidney injury presented in the preconditioned group was cell edema.

Microscopic examination of the liver revealed in both groups only moderate vascular stasis and moderate inflammatory infiltrates in the periportal areas.

\section{Discussion}

In this study a biological, pathological and functional IRI assessment was performed on both local and systemic levels. Local injuries secondary to acute ischemia of the hind limb revealed significant rhabdomyolysis in the control group compared to the ischemic preconditioned group. CK and myoglobin values presented also a statistically significant increase, although maximum values did not exceed more than 3-4 times normal values. Hypoxic and ischemic cell injury secondary to impaired blood flow was followed by impaired oxidative phosphorylation. The decrease of mitochondrial ATP deposits induces $\mathrm{Na}^{+}-\mathrm{K}^{+}$pump dysfunction with $\mathrm{K}^{+}$ion efflux and $\mathrm{Na}^{+}$ions and water influx.

The direct consequence was the onset of cellular edema and structural changes. Increased influx of $\mathrm{Ca}^{2+}$ ions, the loss of glycogen reserves and the alteration of protein synthesis can determine organ dysfunction, however at this stage, alteration are reversible if blood flow is restored $[10,11]$.

Reperfusion generates more severe tissue injuries compared to those induced by ischemia, while ischemic tissues releases significant quantities of toxic oxygen radicals. Both toxic oxygen radicals and super oxides induce endothelial injury, increased vascular permeability (tissue edema during reperfusion) and trigger inflammatory response. Activation of neutrophils, adhesion molecules and cytokines are responsible for the onset of reperfusion injury. While tissue ischemia is the main trigger of pathophysiological changes, reperfusion is the condition that triggers the inflammatory response [7].

IP is the most powerful mechanism against IRI as it improves molecular and cellular cascade and alleviates acute lung and tissular injuries $[1,12]$.

After a period of ischemia, tissues adapt to anaerobic metabolism. The restoration of blood flow will induce an excessive supply of oxygen, thus activating the macrophages in the blood vessels and generating superoxide radicals and ROS, which will in turn induce oxidative stress [7].

IP improves muscle tissue perfusion and stimulates a decrease in blood flow to tissue following ischemia-reperfusion injury of the skeletal muscle [13]. Our study also demonstrated that ischemic lesions of the skeletal muscle were more frequent in the control group compared to the IP group represented by diabetic rats. This was expressed by elevated myoglobin and CK levels. IP increased adenosine levels and the number of $\mathrm{K}^{+}$- ATP channels, improving the oxygen supply and increasing lactate removal [14].

The augmentation of adenosine level might explain why rhabdomyolysis is less severe in the diabetic ischemic preconditioned rats. Our study is in agreement with a previous study of Harkin et al who found fewer skeletal muscle necrosis in IP rats than in healthy subjects [12].

Systemic effects of IRI were also mitigated in the group of preconditioned rats. Przyklenk et al [15] described for the first time remote ischemic preconditioning (RIP), demonstrating that the short-term occlusion of the coronary arteries may have protective effect on the ischemic heart, effects subsequently observed in other organs too (liver, kidney, lungs).

The assessment of renal function was performed by monitoring urea and creatinine values. Although renal impairment was more severe in group 2 ( $1.5 \mathrm{x}$ normal values) than in the control group, it did not reach statistical significance. The increase of urea was higher in the diabetic rats, secondary to dehydration syndrome, due to osmotic diuresis.

Histopathological examinations highlighted more significant structural alterations in the control group. These results are in accordance with the noted biochemical changes. Preservation of the renal function might be explained by early revascularization after acute ischemia, right before the onset of irreversible injuries.

Ischemic-reperfusion injury in the liver induces cholestasis and temporarily reduces bile secretion [16]. The best indicators of IR liver damage are represented by enzyme activitiy and histopathological changes.

Wang et al [16] demonstrated the hepatic protective role of RIP in the first and in the third hour after liver reperfusion injury in mice [7]. In our study, the increase of ALAT and ASAT values in group 2 was statistically significant.

The elevated values of ALAT enzyme were interpreted as a sign of dysmetabolic disorder, because of its hepatic in- 
jury specificity. This interpretation is also supported by the significant hepatomegaly (marker of dysmetabolic injury) present at autopsy. The increase of the ASAT enzyme values might be interpreted as a sign of rhabdomyolysis and dysmetabolic myopathy, rather than hepatic reperfusion injury. Copray et al [17] revealed the presence of muscle injury in diabetic rats, especially of the atrophic type of fast fibers and/or destruction of the sarcoplasmic reticulum, with implicit decrease in muscle mass (rhabdomyolysis being less important).

The biochemical quantification of the degree in tissue injuries was expressed by the LDH value (nonspecific indicator). In our study, LDH values were significantly higher than normal in both groups, suggesting increased accumulation of lactic acid in the tissues and the shift to aerobic metabolism (reperfusion). Since there was no statistically significant difference between the two groups, this can be explained by the fact that IP improves the blood flow by the local and systemic up-regulation of the permeability transition pore, increasing lactate mitochondrial influx and subsequent oxidation [14]. The increased mean value of $\mathrm{LDH}$ in group 2 is due to the fact that the effects of IP on oxidative injury in case of ischemic-reperfusion are more reduced in diabetic rats [18].

The functional assessment of acute ischemia-reperfusion injury of the skeletal muscle did not reveal statistically significant changes between the two studied groups regarding the peak tetanic tension, titanic contraction velocity, specific tension or muscle relaxation velocity. However, in rats, which underwent ischemic preconditioning, the mean values of contraction and relaxation velocity were higher than in the control group and post-contraction muscle relaxation period was shorter. We also noticed a paradoxical response.

The value of the contractile force was increasing during the whole period of the stimulation. In case of ischemia, the slowing down in muscle contraction and relaxation was attributed to higher concentration of hydrogen ions, ADP and the concentration of inorganic phosphate [19]. Hypoperfusion and hypoxia are considered contributing factors in this regard, as their presence is partly dependent on local blood flow and oxygen supply.

De Groot et al [19], and Crisefully et al [20], demonstrated that hind limb IP increases the maximum force during physical exercise after ischemia and reperfusion, due to increased oxidative phosphorylation and accumulation of nitric oxide. Loukogeorgakis et al [21] demonstrated that the effectiveness of IP is directly proportional to the muscle mass subjected to preconditioning and Barbosa et al showed that after each cycle of ischemia/reperfusion a cumulative vasodilatation occurs, because nitric oxide from the skeletal muscle stimulates basal mitochondrial biogenesis [22].

Muscle fatigue is directly dependent on tissue perfusion and clearance of metabolites, especially lactate [22]. Minamino demonstrated that IP and RIP improve capillary flow deficit in case of ischemia-reperfusion [23], reduce the accumulation of lactate in the blood, increase muscle efficiency while using ATP (via ATP sparing), increase mitochondrial flow, mitochondrial biogenesis and thus increase the efficiency in coupling excitation and contraction $[14,22,24]$. This will result in delaying the onset of muscle fatigue, as demonstrated in our study, by improving muscle performance due to ischemic preconditioning in rats and the occurrence of paradoxical response.

\section{Conclusions}

Ischemic preconditioning reduces the severity of reperfusion injury and allows the preservation of muscle contractile function in diabetics after acute ischemia. This subsequently can be considered a favourable prognostic factor.

\section{Acknowledgment}

This article was published in the framework of the European Social Fund, Operational Programme, Development of Human Resources 2007 - 2013, Contract No. POSDRU /19/1.5/S/136893.

\section{Conflicts of interest}

The authors declare that there are no conflicts of interest regarding the publication of this paper.

\section{References}

1. Souza Filho MVP, Loiola RT, Rocha El, et al. Hind Limb Ischemic Preconditioning induces an Anti-inflammatory response by Remote Organs in Rats. Braz J Med Biol Res. 2009;42(10):921-929.

2. Vignaud A, Hourde C, Mejdia F, et al. Impaired Skeletal Muscle Repair after Ischemia-Reperfusion Injury in Mice.Journal of Biomedicine and Biotechnology. 2010;10:10.

3. Zhang B, Zhao Y,Bao L, et al. Protective Effects of Remote Limb Preconditioning on Ischemia reperfusion Injury in Rats Liver. Journal of GHR. 2013;2(9):791-794.

4. Fudickar A, Kunath S, Voß, et al. Effect of Ischemic and Pharmacological Preconditioning of Lower Limb Muscle Tissue on Tissue Oxygenation Measured By Near-Infrared Spectroscopy-A Pilot Study. BMC Anesthesiology. 2014;14:54.

5. Saita Y, Yokoyama K, Nakamura K, Itoman M. Protective Effect of Preconditioning Against Ischemia-Induced Reperfusion Injury of Sckeletal Muscle How Many Preconditioning are Appropriate?. British Journal of Plastic Surgery. 2002;55(3):241-245.

6. Kanoria S, Jalan R, Seifalian AM, Williams R, Davidson BR. Protocols and Mechanisms for Remote Ischemic Preconditioning: a Novel Method for Reducing Ischemia Reperfusion Injury. Transplantation. 2007;84:445458.

7. Uysal Al, Ocmen E, Akan M, et al. The Effects of Remote Ischemic Preconditioning and N-Acetylcysteine with Remote Ischemic Preconditioning in Rat Hepatic Ischemia Reperfusion injury Model. BioMed Research International. 2014; doi: 10.1155/2014/892704.

8. Akbarzadeh A, Norouzian D, Mehrabi MR et al. Induction of Diabetes by Streptozotocin in Rats. Indian J Clin Biochem. 2007;22(2):60-64.

9. Wei M, Ong I, Smith MT, et al. The Streptozotocin-diabetic rat as a model of the Chronic Complications of Human Diabetes. Heart Lung Circ. 2003;12(1):44-50.

10. Macario AJ, Conway de Macario E. Stress and Molecular Chaperones in Disease. Int J Clin Lab Res. 2000;30:49.

11. Chambless L, Folsom AR, Sharrett AR, et al. Coronary Heart Disease Risk Prediction in the Atherosclerosis Risk in Communities (ARIC) study. Journal of Clinical Epidemiology. 2003;56:880.

12. Harkin DW, Barros D'Sa, McCallion K, Hoper M, Campbell C. Ischemic Preconditioning Before Lower Limb Ischemia-Reperfusion Protects Against Lung Injury. Journal of Vascular Surgery. 2002;35(6):1264-1273.

13. Jerome SN, Akimitsu T, Gute DC, Korthuis RJ. Ischemic Preconditioning Attenuates Cappilary No-reflow Induced by Prolonged Ischemia and 
Reperfusion. Am J Physiol. 1995;286:2063-2067.

14. Bailey TG, Jones H, Gregson W, et al. Effect of Ischemic Preconditioning On Lactate Accumulation and Running Performance. Medicine\&Science in Sports \& Exercise. 2012;44:2084-2089.

15. Przyklenk K, Bauer B, Ovize M, Kloner RA, Whittaker P. Regional Ischemic „Preconditioning” Protects Remote Virgin Myocardium from Subsequent Sustained Coronary Occlusion. Circulation. 1993;87(3):893-899.

16. Wang F, Birch SE, He R et al. Remote Ischemic Preconditioning by Hindlimb Occlusion Prevents Liver Ischemic/Reperfusion Injury: the Role of High mobility Group-box 1. Annals of Surgery. 2010;251(2):292-299.

17. Copray S, Liem R, Brouwer N, et al. Contraction-Induced Muscle Fiber Damage is Increased in Soleus Muscle of Streptozotocin-Diabetic Rats and is Associated with Elevated Expression of Brain-Derived Neurotrophic Factor mRNA in Muscle Fibers and Activated Satellite Cells.Experimental Neurology. 2000;161:597-608.

18. Zhu XH, Yuan HJ, Wu YN, et al. Non-invasive Limb Ischemic Preconditioning Reduces Oxidative Stress and Attenuates Myocardium ischemia-reperfusion Injury in Diabetic Rats. Free Radical Research. 2011;45(2):201-210.
19. de Groot PC, Thijssen DH, Sanchez M, Ellenkamp R, Hopman MT. Ischemic preconditioning improves maximal performance in humans. Eur J Appl Physiol. 2010:108:141-146.

20. Crisafulli A, Tangianu F, Tocco F, et al. Ischemic preconditioning of the muscle improves maximal exercise performance but not maximal oxygen uptake in humans. J Appl Physiol. 2011;111:530-536.

21. Lonkogeorgakis SP, Panagiotidon AT, Broadhead MW, Donald A, Deanfield JE, MacAllister RJ. Remote ischemic preconditioning provides early and late protection against endothelial ischemia - reperfiusion injury in humans: role of the autonomic nervous system. J Am Coll Cardiol. 2005;46(3):450-456.

22. Barbosa TC, Machado AC, Braz ID, et al. Remote Ischemic Preconditioning Delays Fatigue Development during Handgrip Exercise. Scand J Med Sci Sports. 2014; doi:10.1111/sms.12229.

23. Minamino T. Cardioprotection from Ischemia/Reperfusion Injury Basic and Translational Research.Circ J. 2012;76:1074-1082.

24. McConell GK, Wadley GD. Potential Role of Nitric Oxide in ContractionStimulated Glucose Uptake and Mitochondrial Biogenesis in Skeletal Muscle. Proceedings of the Australian Physiological Society. 2008;39:6974. 